\title{
Manejo endovascular en pacientes con malformación vascular renal: revisión de la literatura y la experiencia de 2 casos
}

\author{
Endovascular management in a patient with kidney vascular \\ malformation: review of the literature and the experience of 2 cases
}

Eduardo LLahyah', Gonzalo Ortiz

\section{RESUMEN}

Las malformaciones arteriovenosas (MAV) renales son alteraciones poco comunes que consisten en comunicaciones anómalas entre el sistema arterial y venoso intrarrenal. En su presentación clínica, predominan la hematuria y la hipertensión, observándose deterioro cardíaco o pulmonar en un $5 \%$ de los casos. La hematuria es un motivo de consulta frecuente en los servicios de urgencias; sin embargo, en algunas oportunidades esta no recibe la atención que merece y se corre el riesgo de pasar por alto algunas patologías de importancia. La potencial posibilidad de ofrecer un tratamiento curativo hace muy importante su diagnóstico, permitiendo modificar el curso natural de la patología. La arquitectura vascular heterogénea de cada MAV determina las técnicas de tratamiento endovascular a emplear.

La hematuria macroscópica súbita y anemizante debe ser estudiada con detalle puesto que puede comprometer la vida. La realización de procedimientos mínimamente invasivos permite a los pacientes preservar su función renal con mínimas complicaciones y les evita procedimientos más agresivos como la heminefrectomía. Reportamos nuestra experiencia con el tratamiento endovascular de 2 casos

El primero es el caso de un paciente varón de 43 años, deportista, y el otro es de un mujer de 28 años, ambos sin antecedentes clínicos de importancia previos y con hematuria macroscópica asociada a coágulos que requirieron embolización selectiva como tratamiento.

Palabras claves: malformación arteriovenosa, hematuria, hipertensión arterial, embolización.

\begin{abstract}
Arteriovenous malformations (AVM) remain relatively rare clinical lesions consisting in abnormal shunts between the arterial and venous vascular systems. In its clinical presentation, hematuria and hypertension predominate, with cardiac or pulmonary deterioration observed in $5 \%$ of cases. Hematuria is a reason for frequent consultation in the Emergency Department. Sometimes it is seen as a minor condition, and physicians might overlook some important diseases. The potential possibility of offering a curative treatment makes its diagnosis very important, allowing the natural course of the pathology to be modified. The heterogeneous vascular architecture of each AVM determines the endovascular treatment techniques employed.

Sudden and anemic macroscopic hematuria should be studied in detail since it can be life-threatening. Performing minimally invasive procedures allows patients to preserve their kidney function with minimal complications and avoids more aggressive procedures such as heminephrectomy.

We report our experience with the endovascular treatment of 2 cases. The first is the case of a 43-year-old male athlete, and another case of a 28-year-old woman, both with no previous significant clinical history and with macroscopic hematuria
\end{abstract}

Keywords: renal arteriovenous malformations, hematuria, arterial hypertension, embolization.

Revista Argentina de Cardioangiología Intervencionista 2020;11(2):77-82. https://doi.org/10.30567/RACI/202002/0077-0082

\section{INTRODUCCIÓN}

Las malformaciones arteriovenosas (MAV) renales son alteraciones poco comunes que consisten en comunicaciones anómalas entre el sistema arterial y venoso intrarrenal ${ }^{1}$.

Las MAV se clasifican en congénitas, idiopáticas y adquiridas, constituyendo las primeras el $25 \%$ del total ${ }^{3-5,7}$. Son más frecuentes entre la tercera y cuarta décadas de la vida, en mujeres y en el riñón derecho ${ }^{1,4,6}$. Las adquiridas, también denominadas FAV (fístulas arteriovenosas), son las más comunes (75\%) y se producen como consecuencia de un trauma iatrogénico, como ser biopsias renales, litotricia extracorpórea o cirugía. La prevalencia estimada de las MAV renales es inferior al $0,04 \%^{5,6}$.

Se presentan clínicamente con hematuria micro- o macroscópica en el 75\% de los casos debido a la rotura del vaso displásico dentro del sistema colector urinario. La hematuria es

\footnotetext{
1. Servicio de Hemodinamia. Sanatorio Los Alerces. Rosario. Argentina $\triangle$ Correspondencia: Eduardo LLahyah. eduardo.Ilahyah@gmail.com Los autores declaran no tener conflictos de intereses.
}

Recibido: 27/04/2020 | Aceptado: 15/05/2020 un motivo de consulta frecuente en los servicios de urgencias y en la consulta urológica general. Las causas de la hematuria son múltiples y para su estudio es útil dividirlas en nefrológicas y no nefrológicas; dentro de las últimas se encuentran los tumores renales, la litiasis renal, las infecciones urinarias, los procesos quísticos, los traumatismos urológicos, la radiación, los trastornos metabólicos, las discrasias sanguíneas, los procesos vasculorrenales y la hematuria posvacuo; dentro de las nefrológicas se encuentran las primarias (glomerulopatías, glomerulonefritis) y las secundarias (lupus eritematoso sistémico, púrpura de Schonlein-Henoch, síndrome de Goodpasture, las vasculitis, síndrome de Fabry, microangiopatía trombótica, endocarditis, sepsis y amiloidosis) ${ }^{9}$.

Otras formas de presentación de las MAV renales pueden ser dolor lumbar tipo cólico y en flanco, debido a la acumulación de coágulos en la vía urinaria, náuseas, vómitos, hipertensión arterial (HTA), insuficiencia cardíaca congestiva, soplo continuo o, menos específico, masa palpable en flanco ${ }^{1,3-5,7,8}$.

La angiografía por tomografía computarizada (angio-TAC) es sensible para la detección de MAV renales, mientras que la arteriografía renal selectiva revela la arquitectura vascular en detalle, así como la hemodinámica ${ }^{2,10,12}$. Así pues, el tratamiento se enfoca según la etiología.

La embolización arterial renal (EAR) es el tratamiento pre- 


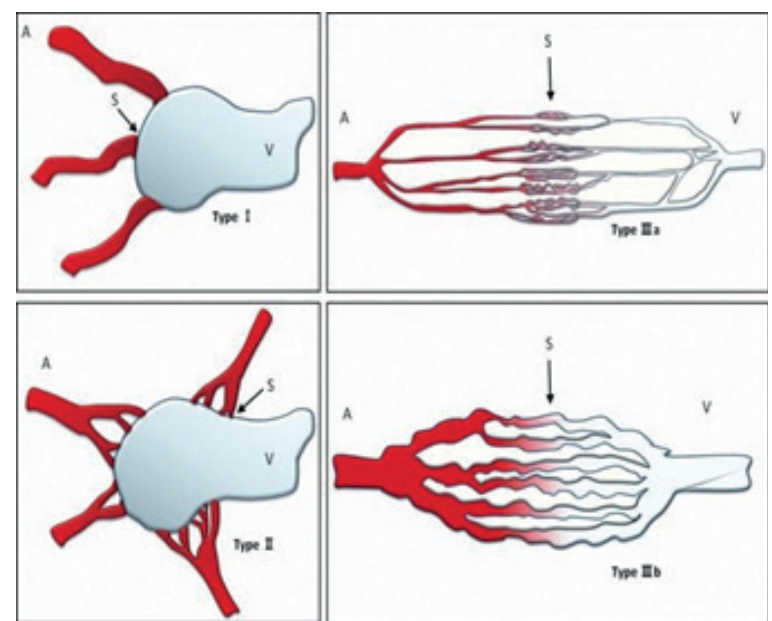

Figura 1. Esquema de la clasificación angiográfica de malformaciones AV por Cho et al. (3): tipo I, que muestra menos de cuatro arterias separadas que se desvían a una sola vena drenante; tipo II, que muestra múltiples arteriolas que derivan a una sola vena de drenaje; tipo IIla, que muestra múltiples fístulas arteriolovenosas finas; tipo IIIb, que muestra múltiples fístulas AV dilatadas que aparecen como una red vascular compleja. A: compartimento arterial de la unidad de fístula. V: compartimento venoso de la unidad de fístula. S: derivación.

ferido para MAV renales con diversos materiales embólicos y ha demostrado buenos resultados. Se presenta el resultado exitoso del manejo de la hematuria mediante la embolización arterial selectiva en dos pacientes con hematuria y sin antecedentes clínicos de relevancia.

\section{REVISIÓN DE LA LITERATURA}

\section{Hallazgos angiográficos}

Las MAV periféricas se clasifican por la escala Cho et al. (C) ${ }^{11}$ dependiendo de la angioarquitectura formada entre las arterias nutritivas, el nido y las venas de drenaje. Esta arquitectura en la angiografía se toma como base para las decisiones terapéuticas y pronósticas. Cho describió 4 arquitecturas distintas (Figura A) y demostró que las MAV con múltiples ramas arteriales que fluyen hacia una sola vena, los tipos I y II, tienen una mejor respuesta al tratamiento. Las AVM con múltiples entradas y salidas, tipos IIIa y IIIb, tienen la peor respuesta.

Yakes $^{26}$ desarrolló una nueva clasificación (Figura 2) de la angioarquitectura de estas lesiones. Este nuevo sistema de clasificación MAV se utiliza para determinar los enfoques endovasculares y los agentes embólicos que serán capaces de eliminar estas MAV. La clasificación de Yakes incluyó lesiones que según la clasificación ISSVA (International Society for the Study of Vascular Anomalies) de 2018 se agrupan como FAV y no como MAV.

\section{Técnica de embolización}

Se accede habitualmente a la arteria femoral común derecha con una vaina $5 \mathrm{Fr}$. El cateterismo selectivo de la arteria renal se realizó con un catéter 5 Fr (Cobra, Cook) para la arteriografía renal. La tasa de inyección de contraste para el angiograma renal se estableció típicamente en 5 a $7 \mathrm{ml} / \mathrm{s}$ con un volumen total de 10 a $15 \mathrm{ml}$. Definimos los MAV renales de alto flujo como la vena de drenaje opacificada antes de la tinción del parénquima renal; de lo contrario, se definió como MAV renales de bajo flujo.

Se introduce un microcatéter de 2,8 Fr, en nuestro caso elegimos (Progreat', Terumo, Japón) en vasos diana identificables para la embolización transarterial.

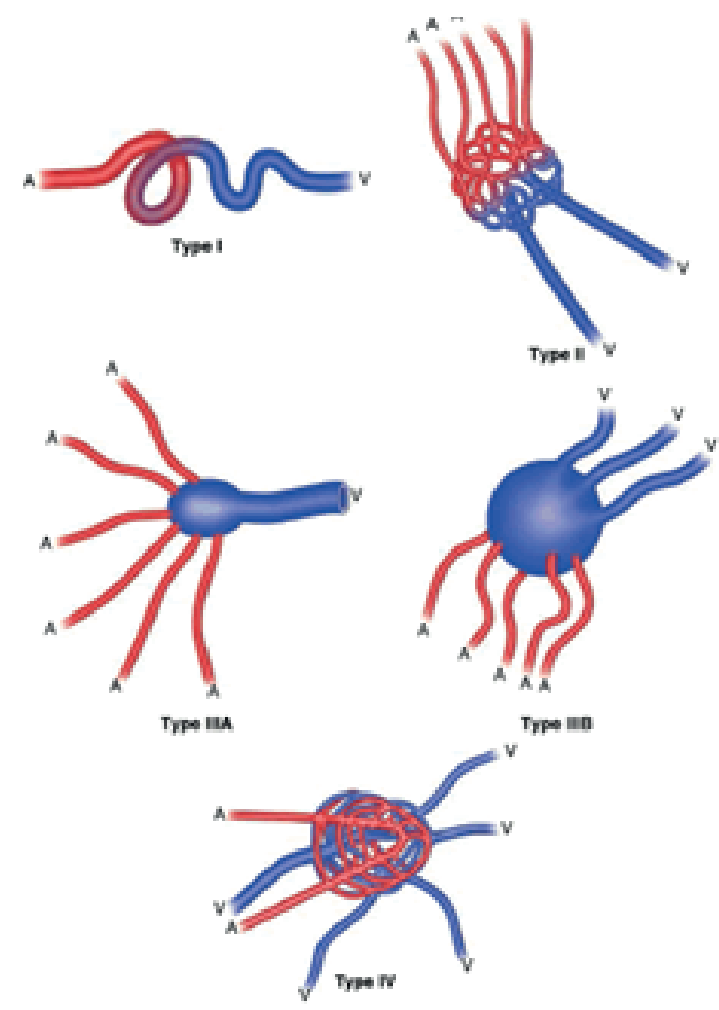

Figura 2. Dibujo esquemático de la clasificación de Yakes. Descripto en Yakes et al. ${ }^{26}$ Tipo I, fístula arteriovenosa directa. Es una conexión directa de arteria a vena. Tipo Ila, múltiples arterias / arteriolas conectadas a un típico"nido" interconectando estructuras tubulares vasculares que luego drenan a venas de flujo de salida. Tipo Ilb, igual que el tipo Ila, excepto que el "nido" drena en una sola vena aneurismática de salida. Tipo Illa, múltiples arteriolas de entrada que derivan a una vena aneurismática de salida. Las fístulas están en la pared de la vena. Tipo IIIb, múltiples arteriolas de flujo de entrada que derivan en una vena aneurismática con múltiples venas de drenaje. Las fístulas (nidos) están en la pared de la vena. Tipo IV, múltiples arterias / arteriolas que se ramifican en forma de paso para formar innumerables microfístulas que infiltran difusamente el tejido afectado. Debido a que el tejido es viable y no está desvitalizado, los lechos capilares también deben estar presentes mezclados entre las microfístulas que drenan a innumerables conexiones venosas.

Se han utilizado varios materiales embólicos para la embolización de MAV renales, incluidas partículas (partículas de esponja de gelatina) y partículas de alcohol polivinílico (PVA), coils metálicos o bobinas (bobinas extraíbles y empujables), tapones vasculares, globos desmontables y materiales líquidos: etanol absoluto, n-butil-cianoacrilato (NBCA) y copolímero de etileno y alcohol vinílico (Onyx). La selección adecuada del material embólico se basa en el tipo de MAV renal y el tamaño y flujo de la fístula.

Después del procedimiento, se realizó una arteriografía renal final para evaluar la oclusión de los vasos diana y el área renal infartada del riñón afectado.

El éxito técnico se definió como la oclusión completa de las arterias de alimentación y el nido de la MAV renal con la mayor preservación las ramas arteriales renales normales en la medida de lo posible. El éxito clínico se definió como la resolución de la hematuria o la desaparición de los síntomas relevantes para MAV.

\section{REPORTE DE CASOS}

\section{Caso clínico 1}

Paciente de sexo masculino de 43 años, deportista (ciclista), de la ciudad de Paraná (Entre Ríos), sin antecedentes clí- 


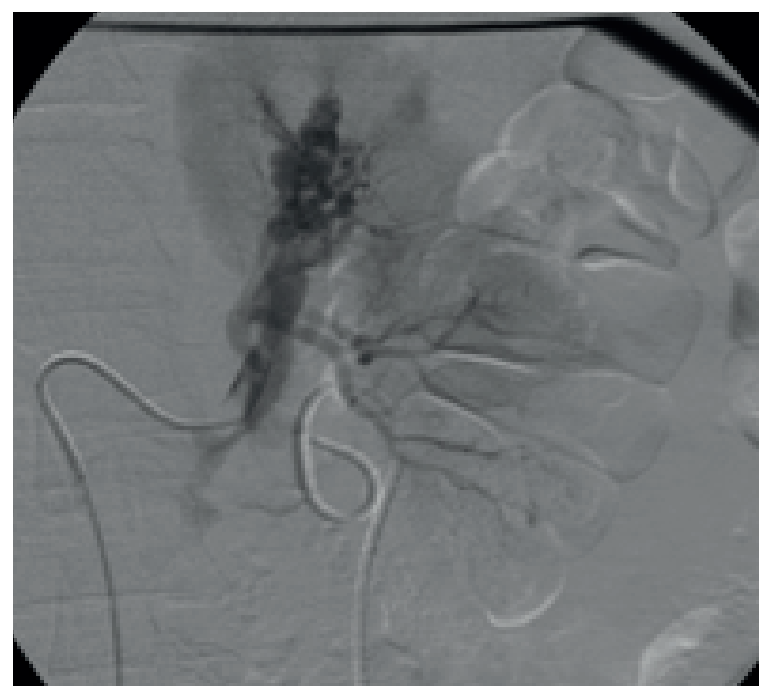

Figura 3.

nicos de importancia. Comienza hace 3 meses de forma repentina con hematuria macroscópica luego de realizar esquí acuático, que no requirió internación ni transfusión, presentando luego astenia, decaimiento y episodios reiterados de hematuria. Se indican exámenes de laboratorio evidenciando recuento Hto 31,2, hemoglobina 11,9, resto de los parámetros normales. La ecografía renal, TAC de abdomen y pelvis y RMN (resonancia magnética nuclear) de abdomen logran identificar una imagen compatible con MAV renal en el polo superior del riñón izquierdo. Se decide realizar una arteriografía renal selectiva en su lugar de origen, que informa MAV renal con fístula de alto flujo (Figuras 3 y 4). Se realiza un ateneo multidisciplinario donde se indica como tratamiento embolización arterial renal selectiva.

Luego fue derivado por su empresa de medicina prepaga a nuestro centro donde se le realizó la embolización superselectiva de MAV renal con una mezcla de NBCA (Histoa$\mathrm{cryl}^{\circ}$ ) y aceite yodado (Lipiodol; Guerbet ( (Figura 5). Para ocluir completamente el nido se requirió sacrificar una arteria segmentaria, lo que indujo un infarto renal pequeño (Figura 6). Como complicación del procedimiento, el paciente presentó leve molestia en el costado del lado afectado, que desapareció en 3 días. El paciente fue dado de alta al 2 do día posprocedimiento, luego de realizarse una TAC de control que mostraba la MAV embolizada y la zona de infarto renal en polo superior.El análisis de laboratorio presentó función renal normal.

Se realiza seguimiento a los 3 y 6 meses con uro-TAC de abdomen y pelvis (Figura 7, corte axial sin contraste ev; y Figura 8, corte coronal con contraste ev) y laboratorios con función renal, evolucionando con perfecto estado de salud y sin recidivas de hematuria.

Mujer de 25 años de edad, quien consulta al servicio de urgencias por cuadro clínico de un día de evolución consistente en dolor hipogástrico y hematuria franca con coágulos de inicio súbito, sin antecedentes personales de importancia; al examen físico se encontró en buenas condiciones generales, estable hemodinámicamente, leve palidez en mucosas, abdomen blando, no doloroso, sin masas ni signos de irritación peritoneal, el resto del examen físico dentro de límites normales. Se interna a la paciente y se tomaron laboratorios que mostraron hemoglobina: 12,7 , hematocrito 37 , leucocitos

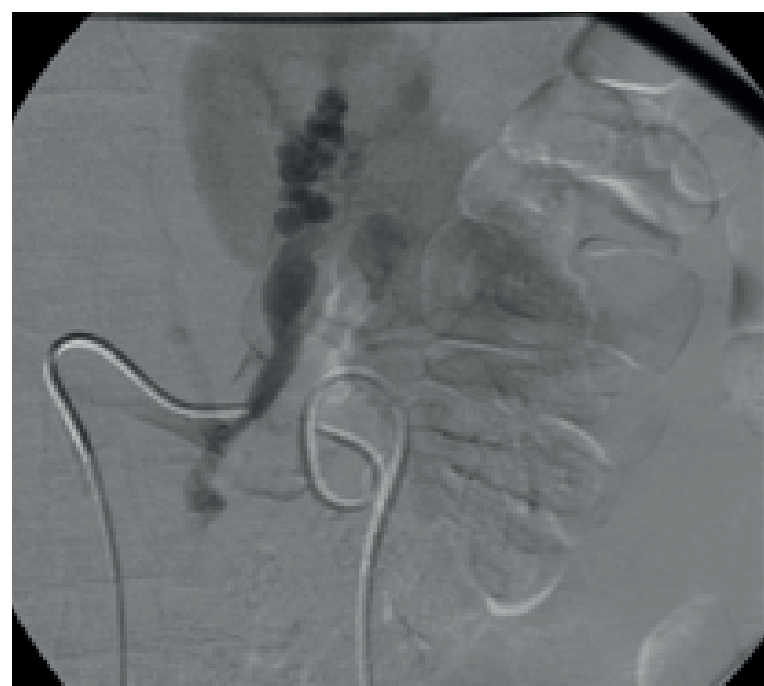

Figura 4.

6800, plaquetas 301.000, tiempos de coagulación normales, citoquímico de orina con $\mathrm{pH} 7$, densidad 1,015, eritrocitos en el sedimento $>20$, proteínas 500 , creatinina 0,7 , PCR negativa, electrolitos normales, ecografía renal y de vías urinarias con coágulos intravesicales. Se realizó urotomografía que evidenció coágulos intravesicales e imagen en grupo calicial superior de densidades heterogéneas compatible con coágulo, además con retardo en la eliminación del contraste en riñón derecho; el servicio de Urología le realizó uretero-renoscopia flexible diagnóstica donde se encontró hematuria lateralizante derecha, múltiples coágulos en pelvis renal y cáliz superior, en este último se identificaron lesiones planas, eritematosas, de aspecto vascular, otra lesión cribiforme a través de la cual se observó salida de sangre de forma pulsátil, se coloca catéter JJ.

La paciente persistió con hematuria macroscópica y la hemoglobina cayó a 7,8, asociado a mareos y adinamia, por lo que se transfundieron 3 unidades de glóbulos rojos. Ante la sospecha de una malformación arteriovenosa se realizó arteriografía, que estuvo dentro de límites normales, por lo que se hizo angio-RMN que descartó malformaciones vasculares u otras lesiones en parénquima renal.. Ante la persistencia del cuadro se decidió realizar nueva arteriografía que no mostró cambios con respecto a la previa y, por tanto, se decidió embolizar de forma selectiva con partículas de polivinil alcohol (PVA) (Contour 500-710 micras, Boston Scientific) y partículas reabsorbibles (Gelfoam, Pfizer), arterias lobares e interlobares del lóbulo superior del riñón derecho con lo cual la paciente resolvió la hematuria y la hemoglobina permaneció estable. Fue dada de alta al 5 to día. Se controla con laboratorio con función renal a los 15 días, que resultó normal y se decide retiro del catéter JJ.

\section{DISCUSIÓN}

La MAV renal en general se presenta inicialmente como hematuria y es un desafío terapéutico debido a su ubicación profunda en el parénquima renal ${ }^{14}$.

A la hora de estudiar un paciente con hematuria, el médico cuenta con varias ayudas diagnósticas: citoquímico de orina, urocultivo, citologías urinarias, radiografía simple de abdomen, ecografía renal y de vías urinarias, urotomografia (uroTAC (, urografía excretora, resonancia magnética y estudio 


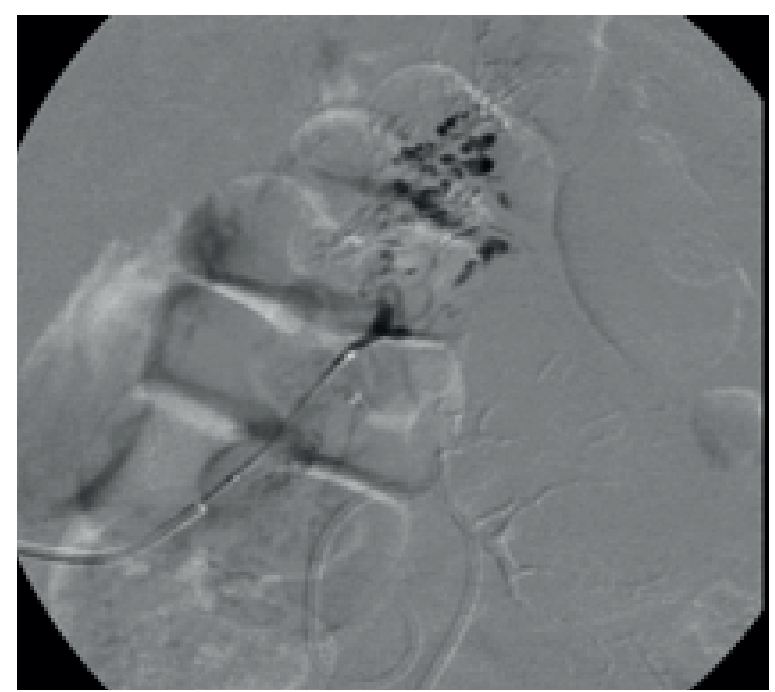

Figura 5. Inyección de n-butil-cianoacrilato $\left(\right.$ Histoacry $\left.l^{\circledR}\right)$ a través de microcatéter en arteria segmentaria.

cistoscópico, siendo este último, en compañía de las citologías urinarias, quizá los más importantes en adultos mayores, pues en ellos se debe descartar como primera causa de sangrado la patología neoplásica, y ofrecen una alta sensibilidad a un muy bajo costo ${ }^{1}$. Sin embargo, no se debe olvidar la importancia de la historia clínica a fin de poder detectar factores de riesgo para patología neoplásica como: tabaquismo, exposición ocupacional a químicos, edad mayor de 40 años, infecciones urinarias recurrentes, abuso de analgésicos y la irradiación pélvica ${ }^{5,6}$.

La hematuria esencial lateralizante, también conocida como hematuria unilateral crónica, hematuria esencial unilateral o hematuria lateralizante benigna, es definida por el hallazgo cistoscópico de hematuria macroscópica en un solo meato, teniendo todas las ayudas diagnósticas imagenológicas normales, como ocurrió en la paciente del caso clínico 2 expuesto. Algunos pacientes pueden presentar cólico como consecuencia del paso de los coágulos, e incluso anemia, pero lo usual es que el paciente sea asintomático; es más común en pacientes jóvenes y no

tiene ninguna predilección por algún género o un lado en particular. Debe realizarse un estudio completo con pruebas de función renal, extendido de sangre periférica, hemograma, pruebas de coagulación, cultivos de orina, citologías urinarias, cultivos para hongos y tuberculosis ${ }^{7}$.

Aún no hay un consenso en cuanto a la realización de arteriografía en todos los pacientes con hematuria esencial, puesto que una malformación arteriovenosa espontánea es rara, así que su realización debería limitarse a pacientes sometidos previamente a biopsia renal, cirugía, trauma renal o tumores renales; también es aceptable realizar una ecografía con Doppler, tomografía contrastada o una angiorresonancia; si el paciente tiene una alta sospecha de malformación arteriovenosa se le debe ofrecer una arteriografía selectiva con embolización $^{8,13}$; son raras las malformaciones arteriovenosas congénitas o idiopáticas, pues lo usual es que estas sean secundarias a procedimientos renales percutáneos como la biopsia renal o los traumas renales, situaciones en las cuales la malformación más común son los pseudoaneurismas ${ }^{10}$.

Las fístulas arteriovenosas son una comunicación anormal entre la circulación arterial y venosa sin tener un colchón capilar, pueden ser congénitas, idiopáticas o iatrogénicas. En la mayoría de los casos las fístulas son clínicamente indetec-

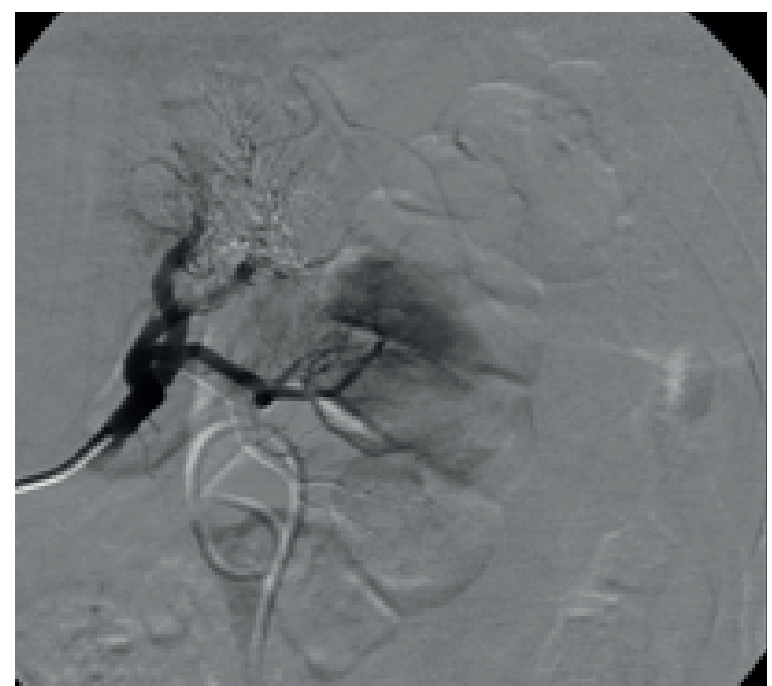

Figura 6. Control posembolización.

tables, sin embargo, pueden causar hematuria macroscópica anemizante, dolor abdominal, hipertensión y, en casos extremos, falla cardíaca e incluso la muerte. Es posible que las fístulas se manifiesten a la par de los aneurismas, lo cual puede ocurrir cuando el hematoma periarterial del aneurisma traspasa la pared del vaso y establece una comunicación entre los dos tipos de circulación ${ }^{11}$. Usualmente las fístulas arteriovenosas son adquiridas luego de traumas o procedimientos percutáneos, aunque pueden existir fístulas idiopáticas que por lo general son solitarias y son consecuencia de los aneurismas renales arteriales congénitos desarrollados al lado de un vaso venoso; su diagnóstico generalmente es debido a un hallazgo incidental en un estudio imagenológico o como parte del algoritmo diagnóstico de la hematuria ${ }^{15,16}$.

Los pacientes con hematuria secundaria a malformaciones vasculares deben ser manejados de acuerdo con la estabilidad hemodinámica presentada, recordando siempre que es una entidad que puede llevar a los pacientes incluso a la muerte; por tanto, en pacientes inestables o con sangrado activo se debe realizar una reanimación adecuada y alguna intervención de forma urgente, mientras que en los pacientes estables las intervenciones son diferibles y en ellos se pueden realizar otras ayudas diagnósticas para aclarar el caso, si es necesario ${ }^{17}$. Las técnicas endovasculares y las cirugías abiertas preservadoras de nefronas son de elección en este segundo grupo de pacientes, es decir, en pacientes estables o incluso asintomáticos; por su parte, en los pacientes inestables o con anatomías vasculares que hacen imposible el tratamiento endovascular, la nefrectomía simple es la única opción terapéutica ${ }^{16}$. Sin embargo, se debe recordar que algunas malformaciones arteriovenosas pueden resolverse de forma espontánea

sin agregar morbilidad a los pacientes, no se conoce exactamente la razón por la cual esto puede suceder, pero se sugiere que la compresión de la lesión por el hematoma, el vasoespasmo, el edema perilesional y el flujo turbulento ayudan a resolver el cuadro, lo cual es más factible en lesiones pequeñas y únicas ${ }^{18}$.

Aunque la angiografía por sustracción digital es el estándar de oro en el diagnóstico de enfermedad vascular ${ }^{19}$, algunas arterias de alimentación fina de las MAV renales no pueden identificarse claramente en la arteriografía inicial, lo que se hará evidente después de la oclusión de las arterias de 


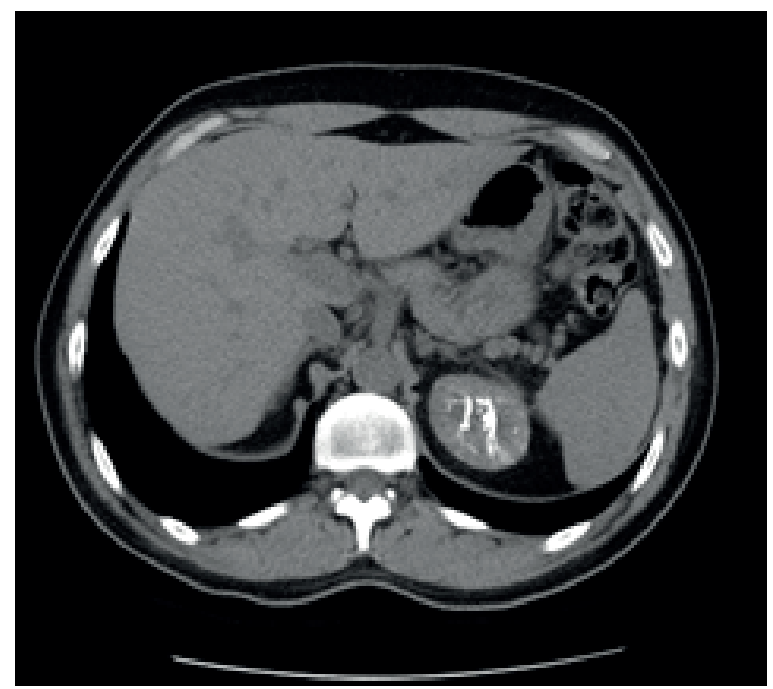

Figura 7. Control a los 4 meses con TAC de abdomen sin contraste ev. Corte axial.

alimentación abierta. Por lo tanto, la arteriografía renal inicial debe realizarse con gran aumento para revelar los detalles de la estructura vascular, y el protocolo de embolización debe modificarse de acuerdo con la arteriografía durante el procedimiento.

La embolización arterial como terapia mínimamente invasiva está siendo empleada desde 1973, antes de esto la única opción terapéutica era la cirugía abierta, con sus múltiples morbilidades y agravantes; en la actualidad, la mayoría de pacientes son intervenidos percutáneamente con grandes tasas de éxito y mínima morbilidad, pues las complicaciones son poco frecuentes, estas incluyen: síndrome posembolización, disección de la arteria renal y pérdida de la función renal como consecuencia de isquemia en el parénquima renal que en ocasiones es más extensa que lo esperado ${ }^{20,21}$, siendo esto último la consecuencia más preocupante por sus implicaciones en la calidad de vida delos pacientes y por tener varios agravantes evidentes como son: el uso de medio de contraste yodado y la desvascularización secundaria a la embolización; sin embargo, esto puede ser disminuido con la embolización superselectiva, pues se minimiza al máximo la pérdida del tejido renal ${ }^{22}$.

En la embolización arterial se pueden utilizar varios materiales tales como: coils metálicos, agentes esclerosantes y material particulado; su selección depende de la anatomía y hemodinamia vascular, además de la patología y el objetivo deseado por el operador.

En los pacientes de los casos clínicos, la embolización se realizó con partículas de Polivinil alcohol (PVA), partículas reabsorbibles (gelfoam) y una mezcla de n-butil-cianocrilato $\left(\right.$ Histoacryl $^{\circ}$ ) con aceite yodado (Lipiodol; Guerbet ( pues el objetivo era obliterar por completo el flujo sanguíneo.

La embolización debe hacerse de forma selectiva, como en estos pacientes, pues así se disminuye el parénquima renal sacrificado por la isquemia al cateterizar una arteria lobar específica, de esta forma los infartos que se causan son segmen-

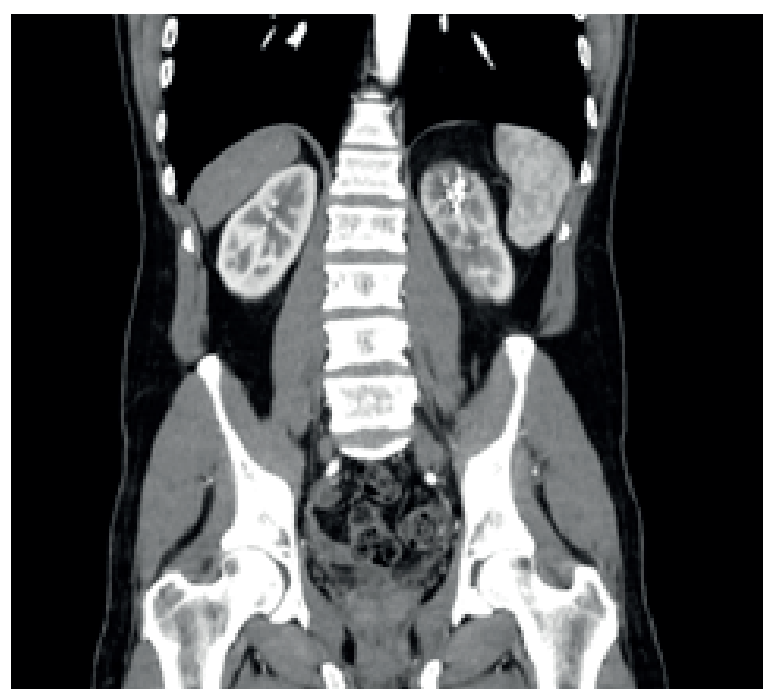

Figura 8. Control con contraste ev. Corte coronal.

tarios y limitados; también pueden embolizarse arterias de forma supraselectiva causando obstrucción específica de ramas arteriales minúsculas, conservando la vasculatura normal alrededor, de esta forma se generan infartos en menos del 10\% del parénquima, lo cual se asocia con escasa reducción en la función renal ${ }^{23}$.

Chatziioannou et al. ${ }^{24}$ presentaron una serie de seis pacientes con malformaciones vasculares renales llevados a arteriografía renal con embolización selectiva con un seguimiento a 23 meses y a quienes se les cuantificó el porcentaje de pérdida de parénquima renal a través de una arteriografía pre- y posembolización, y según mediciones de creatinina pre- y posoperatoria. Todos los pacientes tuvieron desenlaces exitosos y necesitaron una única sesión; en todos la orina aclaró en máximo4 días, todos tuvieron valores de creatinina normales al quinto día cuando fueron dados de alta, excepto un paciente que ya tenía una falla renal instaurada. Ningún paciente presentó complicaciones, el área de isquemia resultante en todos los pacientes fluctuó entre 0 y $30 \%$, con un promedio de $12 \%^{24}$, porcentaje que está demostrado se puede disminuir entre un $0-15 \%$ cuando se realizan embolizaciones supraselectivas ${ }^{25}$.

\section{CONCLUSIONES}

Los pacientes con hematuria macroscópica deben ser estudiados con detalle, porque si bien es claro que la primera causa que se debe descartar son las neoplasias, existen otras causas importantes como las malformaciones vasculares que, de no ser diagnosticadas a tiempo o ser tratadas inadecuadamente, pueden llevar al paciente a la muerte o a la pérdida de la unidad renal. La mejor opción de manejo para estos pacientes debe ser, sin lugar a dudas, la que más beneficios aporte y menos efectos adversos agregue, por tanto, la embolización arterial selectiva se convierte en una muy buena opción, puesto que permite resolver la hematuria y conservar un porcentaje importante del tejido renal. 


\section{BIBLIOGRAFÍA}

1. Hatzidakis A, Rossi M, Mamoulakis C, y cols. Management of renal arteriovenous malformations: a pictorial review. Insights Imaging 2014 Aug;5(4):523-30.

2. Muller A, Rouvière O. Renal artery embolization indications, technical approaches and outcomes. Nat Rev Nephrol 2015 May;11(5):288-301.

3. Dames $E$, y cols. Congenital renal arteriovenous malformation presenting with gross hematuria after a routine jog: a case report. J Med Case Rep 2014 Feb 20;8:65.

4. Coppola D, Costa M, Palazzo C, y cols. Fístula renal arteriovenosa congénita. Tratamiento con embolización selectiva. A propósito de un caso. Rev Arg Urol 2001;67(1):55-8.

5. Zambrana $A R, y$ cols. Arteriovenous renal congenital fistula. Actas Urol Esp 2009 Jun;33(6):696-9.

6. Miranda Utrera N, y cols. Congenital renal arteriovenous malformation: the value of magnetic resonance imaging for diagnosis and intravascular management. Actas Urol Esp 2010 Feb;34(2):215-17.

7. Montoya G, Vega J, Moreno O, Huerta JC. Spontaneous renal arteriovenous fistula-caused hematuria: case report. Gac Med Mex 2004 JanFeb;140(1):85-7.

8. Moreno Alarcón C, et al. Hematuria secondary to congenital arteriovenous fistula treated with embolization. Arch Esp Urol 2011 Jul;64(6):550-3.

9. Sánchez-Carrera F, Leal Hernández F, Moncada Irribarren F, RodríguezFernández E, Díez Cordero JM. Hematuria. En: Urgencias urológicas. Tema monográfico LXI Congreso Nacional de Urología. Madrid: Ene; 1996. pp. 73-83.

10. Maruno, M, Kiyosue, H, Tanoue, S. Renal arteriovenous shunts: clinical features, imaging appearance, and transcatheter embolization based on angioarchitecture. Radiographics 2016;36(2):580-95.

11. Cho SK, Do YS, Shin SW. Arteriovenous malformations of the body and extremities: analysis of therapeutic outcomes and approaches according to a modified angiographic classification. J Endovasc Ther 2006;13(4):527-38.

12. Hwang JH, Do YS, Park KB, Chung HH, Park HS, Hyun D. Embolization of congenital renal arteriovenous malformations using ethanol and coil depending on angiographic types. J Vasc Interv Radiol 2017;28(1):64-70.

13. Campbell-Walsh. Urology, $10^{\circ} \mathrm{ed}$. Evaluation of the Urologic Patient: History, Physical Examination and Urinalysis. Philadelphia: Elsevier; 2012.
14. Crotty KL, Orihuela E, Warren MM. Avances recientes en el diagnóstico y tratamiento de malformaciones arteriovenosas renales y fístulas. J Urol 1993:150(5 Pt 1).

15. Nakada S. Lateralizing Essential Hematuria. Advanced Endourology.The Complete Clinical Guide. Humana Press; 2006.

16. SmaldoneMC, Stein RJ, Cho JS, Leng WW. Giant Idiopathic Renal Arteriovenous Fistula Requiring Urgent Nephrectomy. Urology. 2007Mar;69(3):576. e1-3.

17. Singh D, Gill IS. Renal artery pseudoaneurysm following laparoscopic partial nephrectomy. J Urol 2005;174:2256.

18. Inoue T, Hashimura T. Spontaneous Regression of Renal Arteriovenous Malformation. J Urol 2000;163:232-3.

19. Bookstein JJ, Goldstein HM. Manejo exitoso de la fístula arteriovenosaposbiopsia con embolización arterial selectiva. Radiología 1973;109(3):5356.

20. Schwartz MJ, Smith EB, Trost DW, et al. Renal artery embolization: clinical indications and experience from over 100 cases. BJU Int 2007:99:881.

21. Perini S, Gordon RL, LaBerge JM, et al. Transcatheter embolization of biopsy-related vascular injury in the transplant kidney: immediate and long-term outcome. J Vasc Interv Radiol 1998;9:1011-9.

22. Poulakis V, Ferakis $N$, Becht E, et al. Treatment of renal-vascular injury by transcatheter embolization: immediate and long-term effects on renal function. J Endourol 2006;20:405.

23. Ginat D, et al. Transcatheter Renal Artery Embolization: Clinical Applications and Techniques. Techniques in Vascular and Interventional Radiology 2009;12:224-39.

24. Chatziioannou A, Brountzos E, Primetis E, et al. Effects of Superselective Embolization for Renal Vascular Injuries on Renal Parenchyma and Function. Eur J Vasc Endovasc Surg 2004;28:201-206.

25. Dorffner $R$, Thurnher $S$, Prokesch $R$ et al. Embolization of iatrogenic vascular injuries of renal transplants: immediate and follow-up results. Cardiovasc Intervent Radiol 1998:21:129-34.

26. Yakes WF, Vogelzang $R L$, Ivancev K, Yakes AM. New arteriographic classification of AVM based on the yakes classification system. In: Congenital vascular Malformations 2017: 63-69. 